\title{
Diagnostic features of the Favre-Goldmann syndrome
}

\author{
GERALD A. FISHMAN, LEE M. JAMPOL, AND MORTON F. GOLDBERG \\ From the Department of Ophthalmology, University of Illinois Eye and Ear Infirmary, \\ Chicago, Illinois
}

Traditional descriptions of the Favre-Goldmann syndrome include early onset of night blindness; atypical pigmentary dystrophy of the retina; degenerative changes of the vitreous humour (including both liquefaction and the presence of microfibrillar strands); peripheral and, less often, central retinoschisis; lens opacities; striking electroretinographic (ERG) abnormalities; and autosomal recessive inheritance. We noted previously unreported diffuse leakage from retinal vessels on fluorescein angiography. Electroretinography showed cone and rod responses which we believe are characteristic in the earlier stages of the disease.

\section{Case reports}

\section{CASE I}

A 27-year-old White woman (the proband) was first seen at the University of Illinois Eye and Ear Infirmary in December 1974 for investigation of poor vision in her right eye and decreased night vision for as long as she could remember. Neither symptom had worsened. The patient had no other subjective complaints. She had completely recovered from hepatitis two years previously. The patient was not taking any medications, although she had taken oral contraceptives in the past. Two younger brothers, aged 18 and 22 years, were reported also to have poor night vision. A 16-year-old sister had no ocular complaints. Parental consanguinity was not noted. The case of another younger sister who also complained of poor night vision is described later. Fig. I illustrates this pedigree. The patient's two-year-old daughter and five-month-old son were ophthalmoscopically normal.

The patient's best corrected visual acuity was $20 / 200$ in the right eye with $-4.00 \mathrm{sph}$. $+1.25 \mathrm{cyl}$. $\times 115^{\circ}$, and $20 / 20$ in the left eye with $-1 \cdot 25 \mathrm{sph}$. $+\mathrm{I} \cdot 00 \mathrm{cyl}$. $\times 30^{\circ}$. External examination showed a mild blepharoptosis on the left; the conjunctiva and cornea of both eyes were normal. The patient's right pupil was slightly smaller than the left; no Marcus Gunn phenomenon was demonstrated, and both pupils reacted normally to both direct and consensual light stimuli. The left iris had a more greenish hue than the blue right iris. Motility examination showed a right hyperphoria and slight

Address for reprints: G. A. Fishman, MD, University of Illinois Eye and Ear Infirmary, 1855 West Taylor Street, Chicago, Illinois 60612, USA

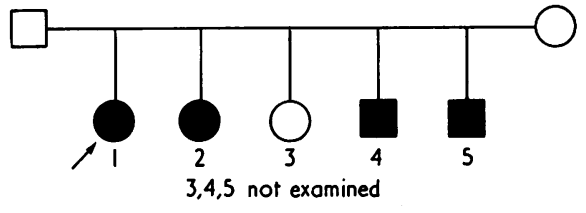

FIG. I Pedigree of cases I and 2. Arrow identifies proband.

overaction of the right inferior oblique muscle. On slit-lamp examination the cornea and anterior chamber were normal. The right lens showed a $\mathbf{I}+$ posterior subcapsular lens opacity while the left lens was clear. The vitreous humour of both eyes was optically empty posteriorly, while anteriorly microfibrillar strands of condensed vitreous were noted. Intraocular pressures were normal in both eyes. Visual field testing showed a symmetrical constriction to within $20^{\circ}$ of fixation with a $4 \mathrm{~mm}$ white test object in the right eye and to within $15^{\circ}$ to $20^{\circ}$ of fixation with a $2 \mathrm{~mm}$ white test object in the left eye.

The right fundus showed extensive peripheral retinoschisis beginning between the equator and ora serrata and extending posteriorly to the superior and inferior temporal vessel arcades. Nasally, the schisis did not extend more than I disc diameter posterior to the equator. Clumping of retinal pigment was seen predominantly in areas of retinoschisis. The schisis and pigmentation were greater temporally than nasally. The optic disc appeared normal. The macula had minimal but definite cystoid changes. In the posterior pole, but not in the macula, subtle, white, thread-like opaque changes were noted within the retinal vessels. As peripheral, more highly raised areas of schisis were approached these opaque changes became more evident in small retinal vessels and often assumed a dendritic appearance (Fig. 2). Similar but less extensive changes were noted in the patient's left eye. In addition to the areas of extensive schisis and opaque vascular changes a full-thickness retinal hole with an operculum was noted at II o'clock near the equator in the left eye. A small vitreous traction band extended from the operculum over the ora serrata.

A dark-adaptation study with a Goldmann-Weekers dark adaptometer showed monophasic curves in both eyes. Final cone thresholds were normal, while a rod component to the dark-adaptation curve was nondetectable. An electro-oculogram (EOG) showed 
subnormal light-peak to dark-trough ratios of $I \cdot 7 I$ and $\mathrm{I} \cdot 62$ in the right and left eyes, respectively. An ERG showed cone responses that were normal in amplitude but much prolonged in implicit time in both eyes (Fig. 3a). During a I 5-minute period of dark adaptation the responses did not change in either implicit time or amplitude. A small $b_{2}$ (probable rod component of the scotopic ERG) was noted (Fig. $3^{b}$ ). A I-4 blue stimulus from a Grass photostimulator was implemented to isolate rod responses and showed a minimal $25 \mu \mathrm{V}$ b-wave deflexion from the baseline while an I-I 6 red stimulus showed only cone ( $x$-wave) responses in both eyes. Flicker fusion frequency testing of both eyes with a high-intensity stimulus showed fusion at 30 cycles/s. Normal fusion frequency rates are $55 \mathrm{cycles} / \mathrm{s}$ or higher.

A fluorescein angiogram accentuated the opaque retinal vessels in the temporal periphery of the patient's right eye. Within areas of most advanced schisis opaque retinal vessels were not perfused (Fig. 4a). In less raised areas delayed perfusion of some remaining opaque vessels was seen (Fig. 4a). Vessel perfusion often appear-

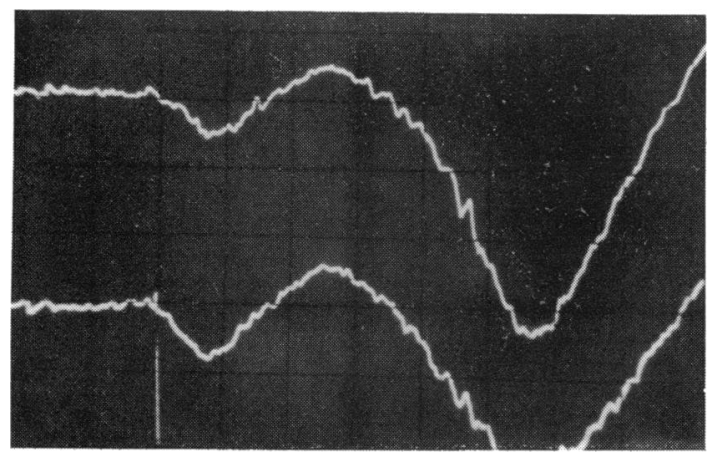

FIG. 3a Case 1. Light-adapted ERG. Both right (upper) and left eye (lower) tracings show prolonged implicit times of $50 \mathrm{~ms}$. Vertical squares represent 100 $\mu V$, horizontal squares $20 \mathrm{~ms}$. Normal cone implicit time, from vertical line, lower left, to b-wave peak is $28 \mathrm{~ms}$

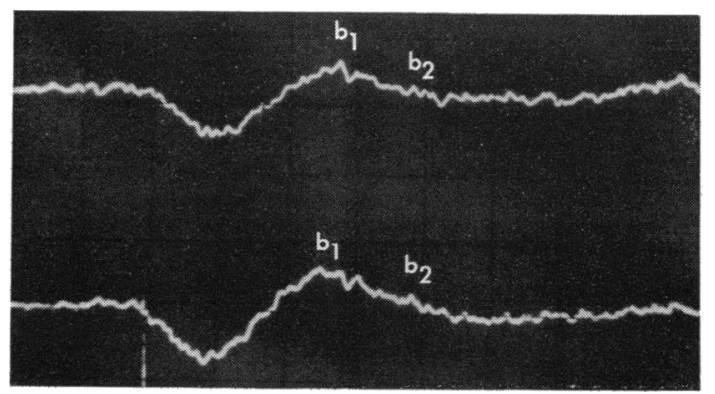

FIG. 3b Case I. Dark-adapted ERG. Note similar amplitudes and implicit times to those in Fig. $3 \mathrm{a}$ : development of $b_{2}$ (rod) portion of ERG scotopic response is only noticeable difference ed to end just posterior to extensive raised areas of schisis (Fig. 4a). Fluorescein leakage occurred from opaque retinal vessels at the margin of perfused and non-perfused retina (Fig. $4 b$ ). These leaking vessels did not represent neovascular tissue. A late-phase fluorescein study of the patient's right macula showed subtle but definite leakage from perifoveal capillaries (Fig. 5) and throughout the posterior pole.

\section{CASE 2}

The 26-year-old sister of case I was seen in January I 975 for investigation of poor night vision since early childhood. The patient's general health was good. Her best corrected visual acuity was $20 / 25$ in the right eye with $+5.75 \mathrm{sph}$. and $20 / 30$ in the left eye with +3.25 sph. +0.25 cyl. $\times 10^{\circ}$. External examination was normal. Versions were full in all directions of gaze. The pupils reacted normally to both direct and consensual light stimuli. Although orthotropic for near, the patient did have a variable 25 to 30 prism dioptre alternating esotropia for distance. Slit-lamp examination of the cornea and anterior chamber was normal in both eyes. There was no evidence of any iris heterochromia. The patient had I + bilateral posterior subcapsular lens opacities and $I+$ cells in the mid-vitreous cavity. The vitreous humour in both eyes appeared optically empty posteriorly while the anterior vitreous showed degenerative microfibrillar strands. On gonioscopy the anterior chamber angles were normal. Fundus examination revealed subtle cystoid changes. The larger retinal vessels appeared to be normal in both calibre and course. An extensive shallow retinoschisis was noted throughout the mid-periphery of both eyes. The schitic changes, which were associated with a moderate amount of black pigment clumping, were present both nasally and temporally. Opaque vessel changes were present throughout the mid-periphery extending anteriorly between the equator and ora serrata (Fig. 6).

Visual field testing with a Goldmann perimeter showed relative symmetrical constriction to within $15^{\circ}$ of fixation in each eye. Results of a dark-adaptation test were similar to those in case $I$ in showing only a monophasic recording with normal final cone thresholds. Colour vision testing with the Farnsworth-Munsell roo-hue test showed a mild blue defect in the patient's right eye and normal colour perception in the left. An EOG showed significantly abnormal light-peak to dark-trough ratios of $\mathrm{I} \cdot 33$ and $\mathrm{I} \cdot 05$ in the right and left eyes, respectively. (Normal values for her age from our laboratory would be $1 \cdot 75$ or greater.) An ERG showed cone responses that were both reduced in amplitude and prolonged in implicit time. After a 20-minute period of dark adaptation there was essentially no change from photopic values in either amplitudes or implicit times in either eye, reflecting an apparent absence of detectable rod activity. Rod responses were also non-detectable with a low-intensity I-4 blue stimulus. Flicker responses with a high-intensity stimulus were non-detectable at 30 cycles/s.

A fluorescein angiogram of the patient's right eye showed extensive leakage from retinal capillaries throughout the posterior pole (Fig. 7). Leakage was present in the mid-periphery in association with vessels 


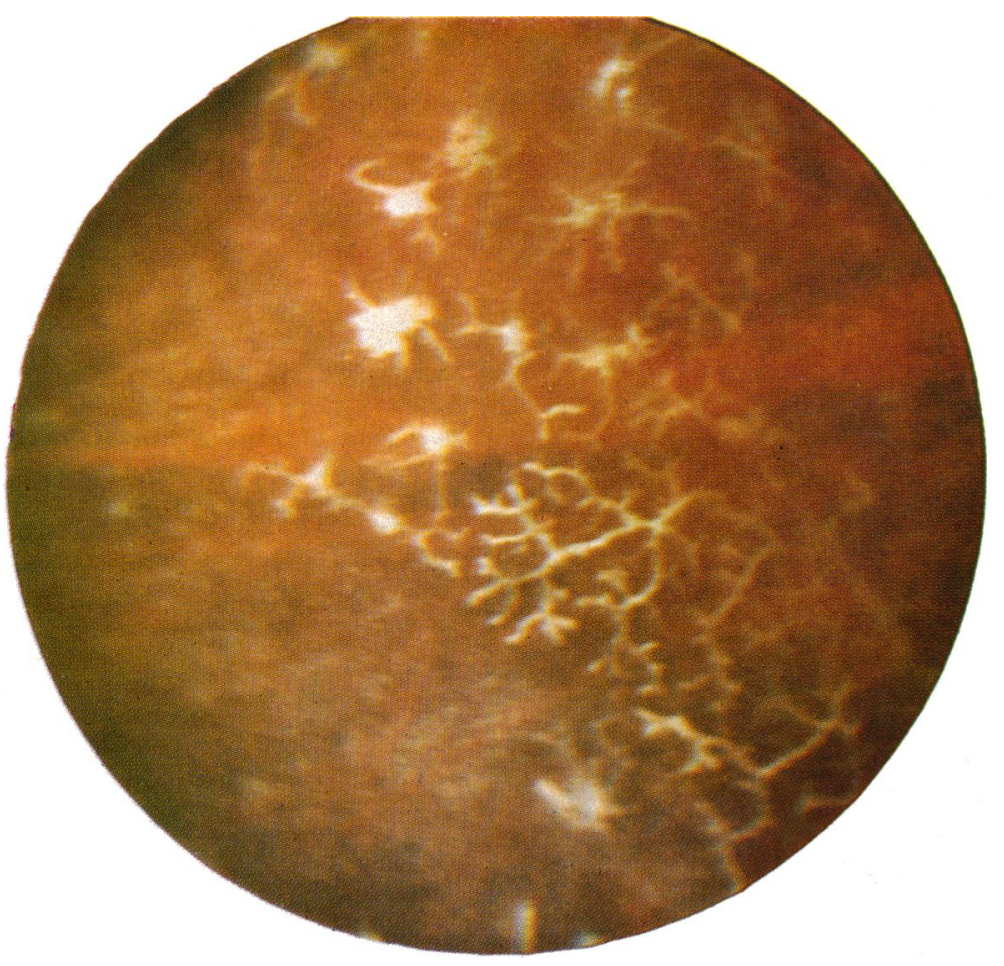

FIG. 2 Case I, right eye. Opaque, dendritic appearance of peripheral vessels

FIG. 6 Case 2. Opaque vessels within mid-periphery of right eye

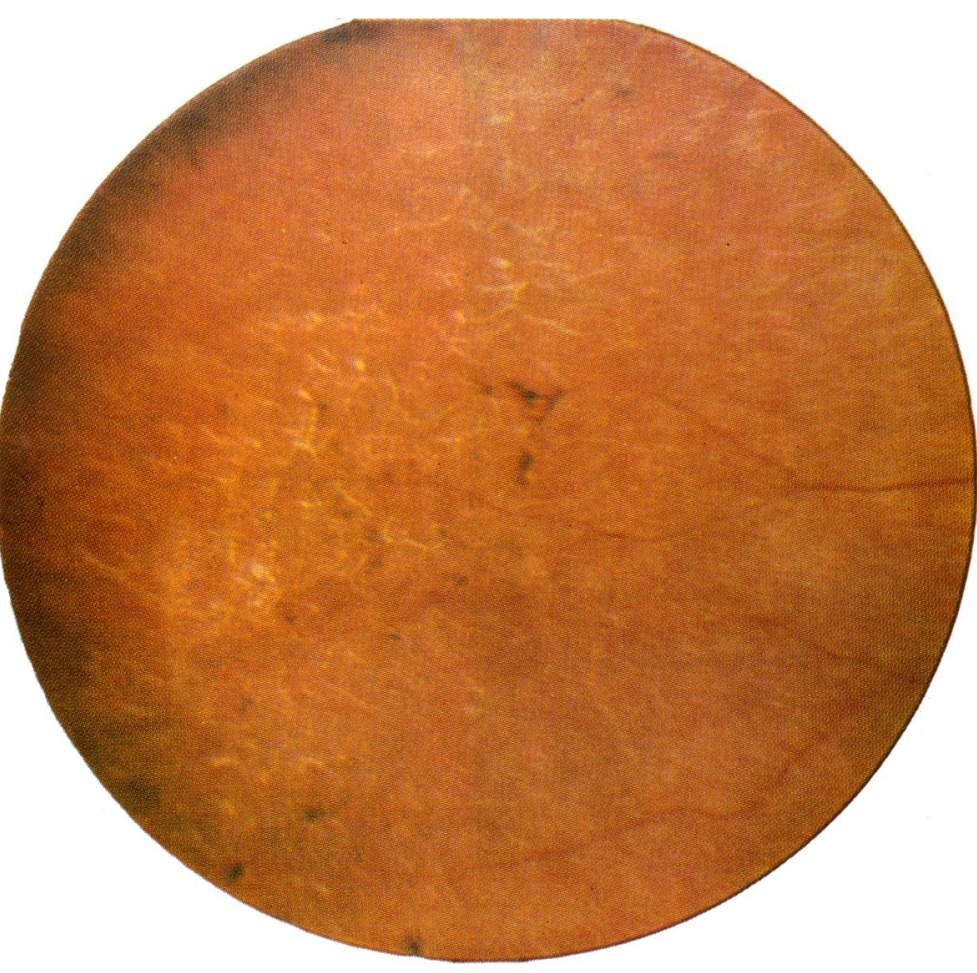




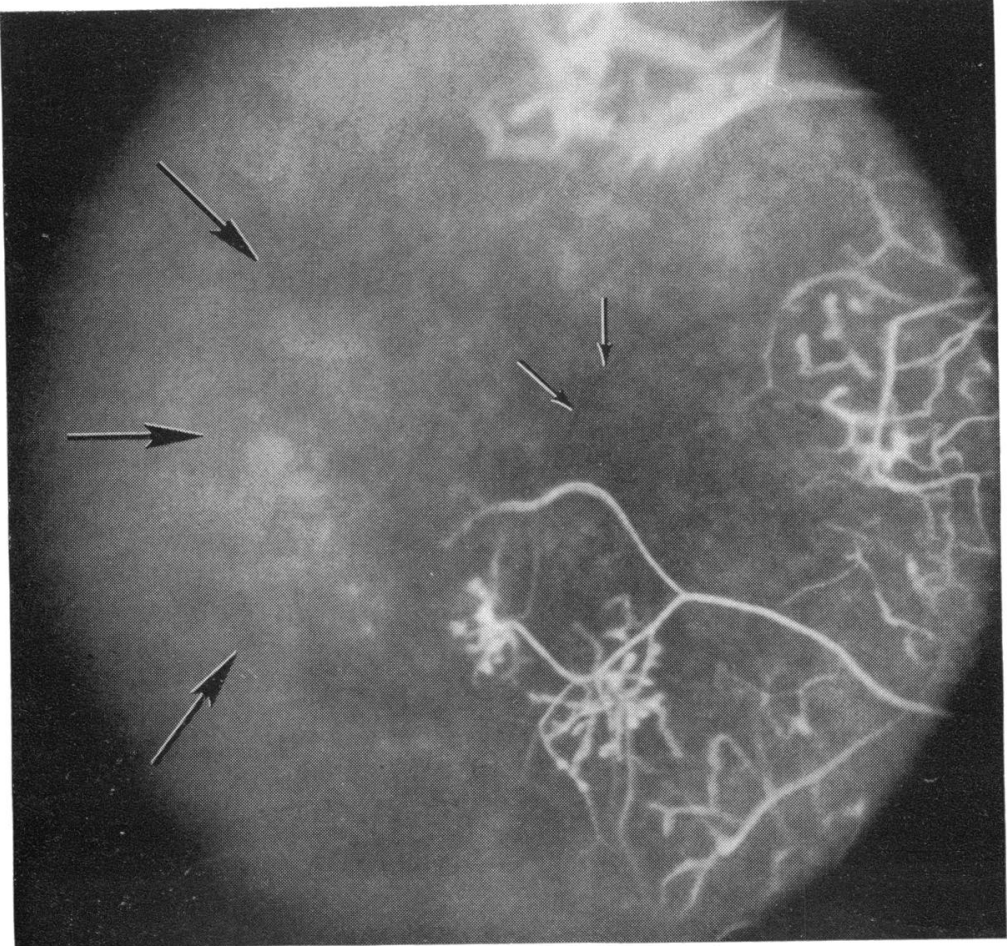

FIG. 4a Case 1. Fluorescein angiogram of temporal periphery from right eye showing opaque, dendritic retinal vessels. Non-perfused retina is noted within areas of marked schisis (larger arrows). Note delayed filling of retinal vessel (smaller arrows)

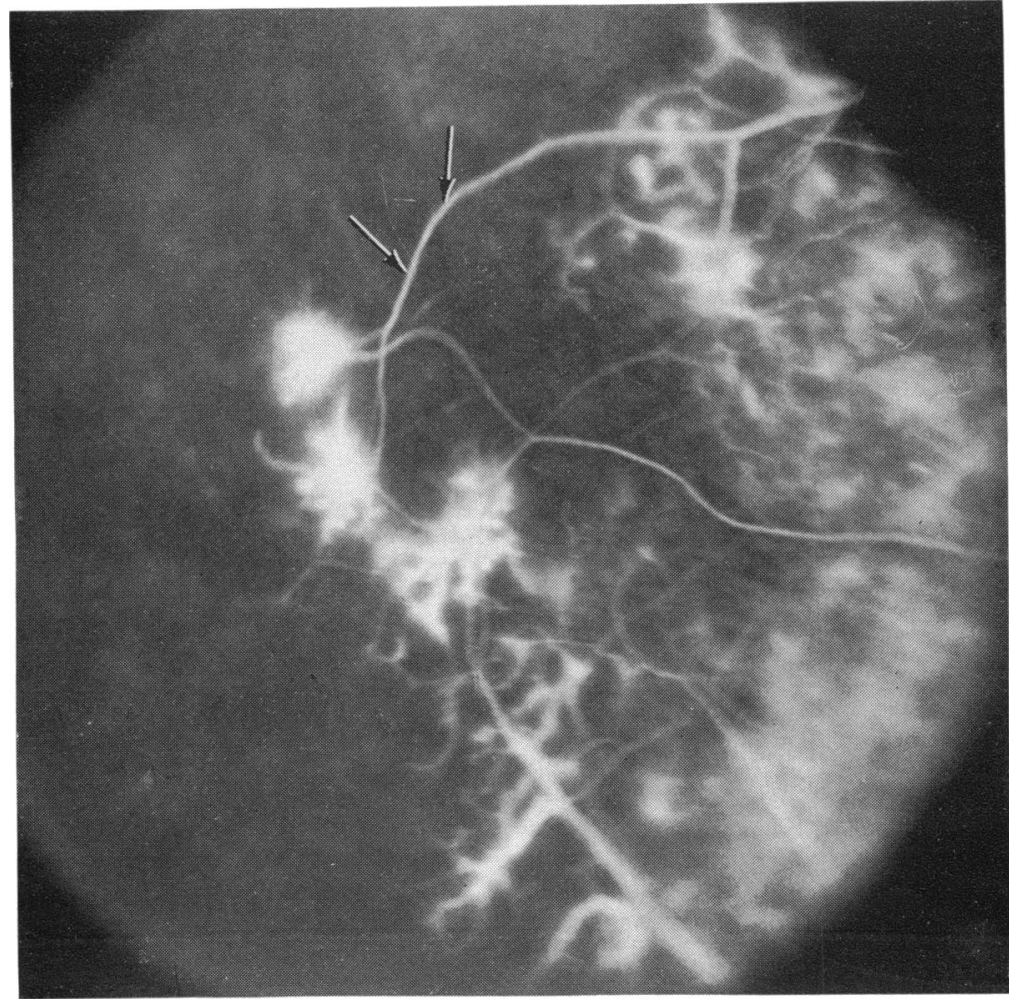

FIG. 4b Leakage of dye from opaque retinal vessels at margin of perfused and non-perfused retina. Note same vessel in Fig. 4a is now perfused (arrows) 
that ophthalmoscopically were opaque. Less leakage occurred in areas where retinoschisis was most evident clinically. Areas of early hyperfluorescence noted in the posterior pole and mid-periphery were associated with retinal pigment epithelial atrophy while spotty regions of hypofluorescence were caused by pigment clumping. In later stages of the angiographic study changes of cystoid oedema were noted in the right macula (Fig. 7).

\section{CASE 3}

A 56-year-old White man, who was unrelated to the patients in cases $I$ and 2, complained of decreased night vision and poor peripheral vision for as long as bilateral $2+$ posterior subcapsular lens opacities. The vitreous humour in both eyes was optically empty posteriorly from extensive liquefaction, while in the anterior vitreous chamber there were spidernet fibrillar strands. Fundus examination showed a bilateral waxytype atrophy of the optic discs and constricted retinal vessels. The foveal reflex was absent in both eyes. Within the superior and inferior temporal mid-periphery of the right eye was a highly raised retinoschisis (Fig. 8). In some areas the schisis extended posteriorly, and ophthalmoscopically appeared to end just posterior to the arcade formed by the large superior and inferior temporal arterioles (Fig. 9). The schisis was progressively less raised posteriorly as it approached the borders of the superior and inferior temporal vessels. All schitic

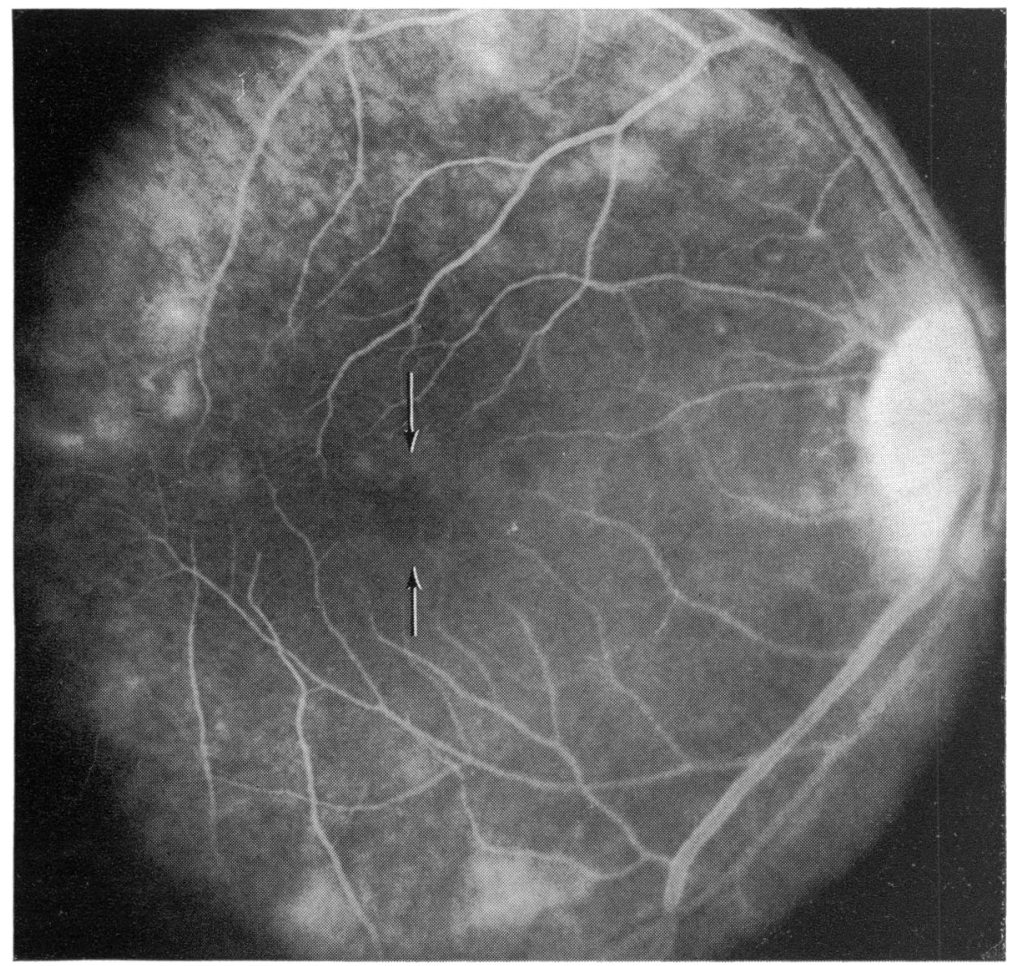

FIG. 5 Case I. Late-phase fluorescein angiogram from the posterior pole. Note leakage from perifoveal (arrows) and other capillaries

he could remember. The central vision in his left eye had also been poor since about age 20 . Vision in the right eye had been good until about 10 years previously, when he began to notice a slow but progressive decrease in acuity. The patient's general health was good. A 55-year-old sister, unavailable for examination, also complained of poor night vision since childhood. Parental consanguinity was not noted.

Visual acuity was $20 / 60$ in the right eye with +6.75 sph. $+\mathrm{r} \cdot 75 \mathrm{cyl} . \times 180^{\circ}$ and $5 / 200$ in the left eye with $+6.00 \mathrm{sph}$. External and motility examinations were normal. Slit-lamp examination of the cornea, iris, and anterior chamber of both eyes was normal. Both pupils reacted normally to direct and consensual light stimuli. Ocular pressure was I I $\mathrm{mmHg}$ in each eye. There were areas were associated with round black clumps of retinal pigment (Figs 8 , 9). On slit-lamp examination of the posterior pole with a contact lens subtle opaque changes were noted within smaller retinal vessels not associated with areas of schisis. These opaque changes became more evident as the vessels approached regions of shallow schisis. At the posterior border of more raised schitic areas the opaque vessels were most evident and appeared dendritic. Although areas of schisis were not noted nasal to the optic disc minimal opaque changes within smaller retinal vessels were also present in these areas. Fundus changes in the left eye were essentially the same as those in the right.

Visual field testing with $4 \mathrm{e}-\mathrm{III}$ and $4 \mathrm{e}-\mathrm{IV}$ isopters on the Goldmann perimeter showed an absolute symme- 


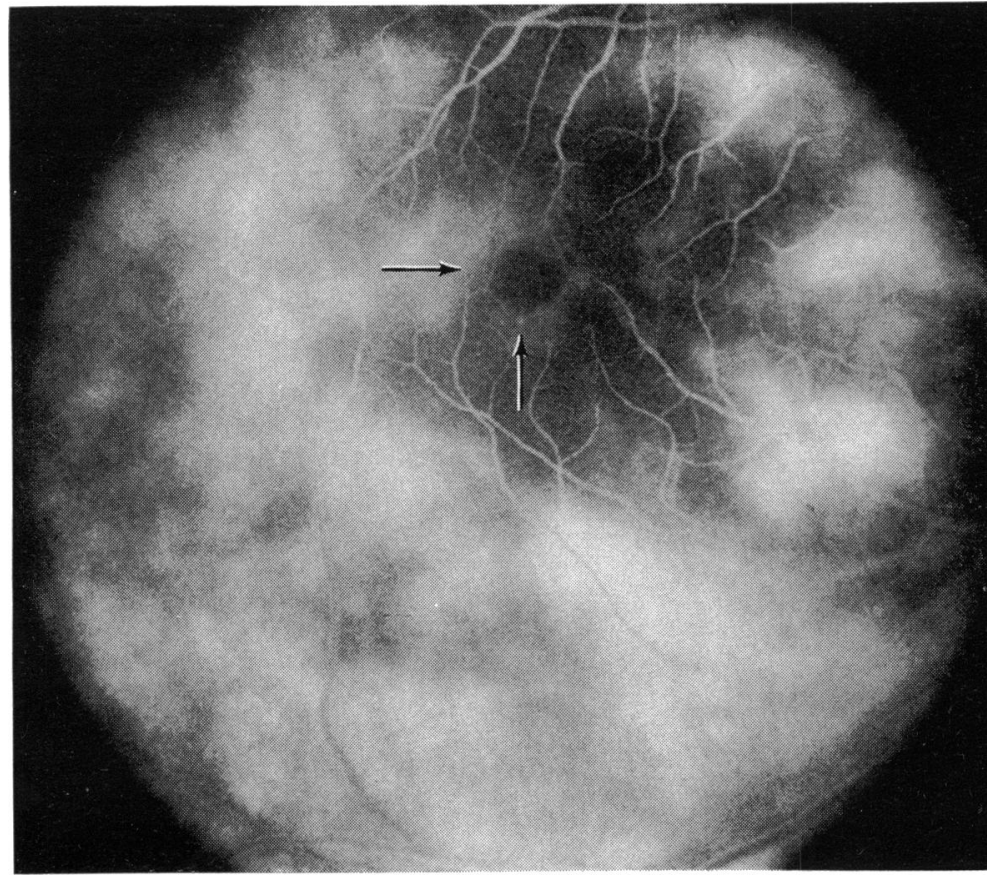

FIG. 7 Case 2. Extensive leakage from retinal capillaries throughout posterior pole. Note leakage from perifoveal capillaries (arrows)

trical constriction to within $15^{\circ}$ of fixation in each eye. Dark-adaption thresholds measured on the right eye showed a monophasic curve with a normal cone threshold. In both eyes ERG responses were non-detectable, while an EOG showed light-peak to dark-trough ratios of $I \cdot I 0$ and $I \cdot I 5$ in the right and left eyes, respectively. On fluorescein angiography leakage from retinal capillaries was evident even in areas which ophthalmoscopically either appeared normal or showed only subtle opaque changes within retinal vessels. Extensive capillary leakage was noted posteriorly in areas of low schisis (Fig. 10). Retinal capillaries were non-perfused in areas where schisis was much raised in the mid-periphery.

\section{Discussion}

Favre (1958) reported vitreoretinal degeneration and the early onset of poor night vision in a 16 year-old boy and his I5-year-old sister. Vitreous changes included both liquefaction and fibrillar degeneration. Fundus changes included equatorial chorioretinal atrophy and pigment clumping. Peripheral and macular retinoschisis was also noted. Ophthalmoscopically the schisis of the macula was reminiscent of microcystic oedema. The boy also had posterior cortical lens opacities. In both cases visual field testing showed annular scotomas, while dark-adaptation studies revealed monophasic curves with normal cone but absent rod thresholds. Their I3-year-old sister was normal, and a paternal grandmother, although not examined by Favre, was reported by the family to have had poor night vision. Ricci (1960) found that ERG responses were non-detectable in Favre's two patients. He reported similar findings in a $4 \mathrm{I}$-yearold woman and emphasized the absence of an ERG response. Ricci suggested an autosomal recessive pattern of inheritance.

In the majority of similar cases subsequently reported (Blanck, Polliot, and Bernard, I973; Carr and Siegel, r970; François, De Rouck, and Cambie, 1974; François and Van Oye, 1967; MacVicar and Wilbrandt, I970; Maraini, I967; Stankovic, Kecmanovic, and Drincic, 1973) ERG responses were non-detectable. Dark-adaptation findings generally included monophasic curves with normal cone thresholds or biphasic curves with much raised rod final thresholds. Recordable ERG responses were noted by Carr and Siegel (1970) in an II-year-old boy with Favre-Goldmann syndrome. The photopic a- and b-waves were described as 'near normal'; a scotopic increment was not observable. No mention was made of implicit time values. The same authors reported an additional case of a 22-year-old man who had a normal ERG photopic and scotopic a-wave but an absence of any positive b-wave response. Since selective absence of an ERG b-wave is characteristic of juvenile $\mathrm{X}$-linked retinoschisis it was not clear from their description how they eliminated this diagnostic possibility.

Recordable but markedly reduced a- and b-wave ERG responses were obtained from a single case 


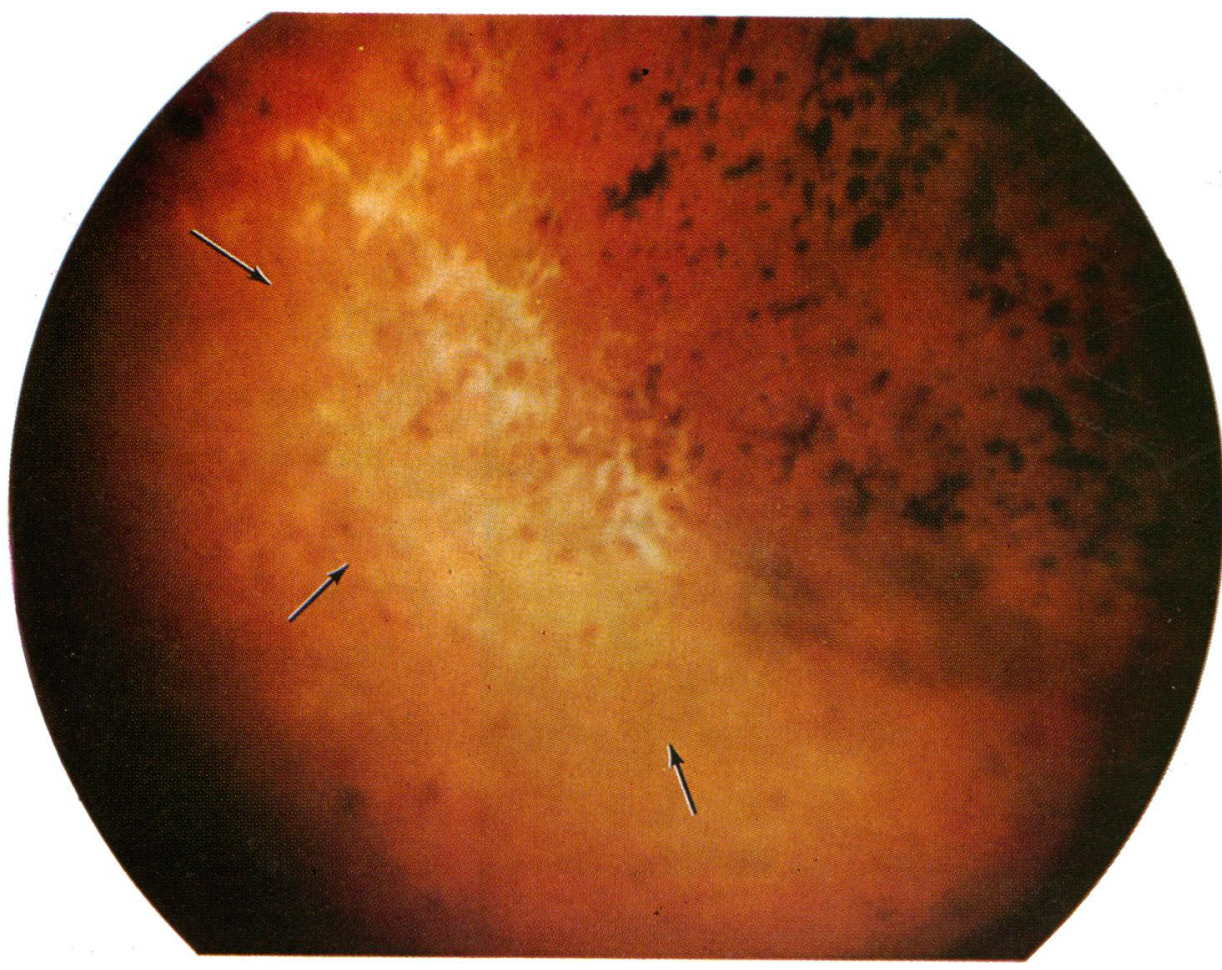

FIG. 8 Case 3.

Highly raised

retinoschisis in

temporal periphery

from right eye

(arrows).

Clumping of

retinal pigment

epithelium is seen

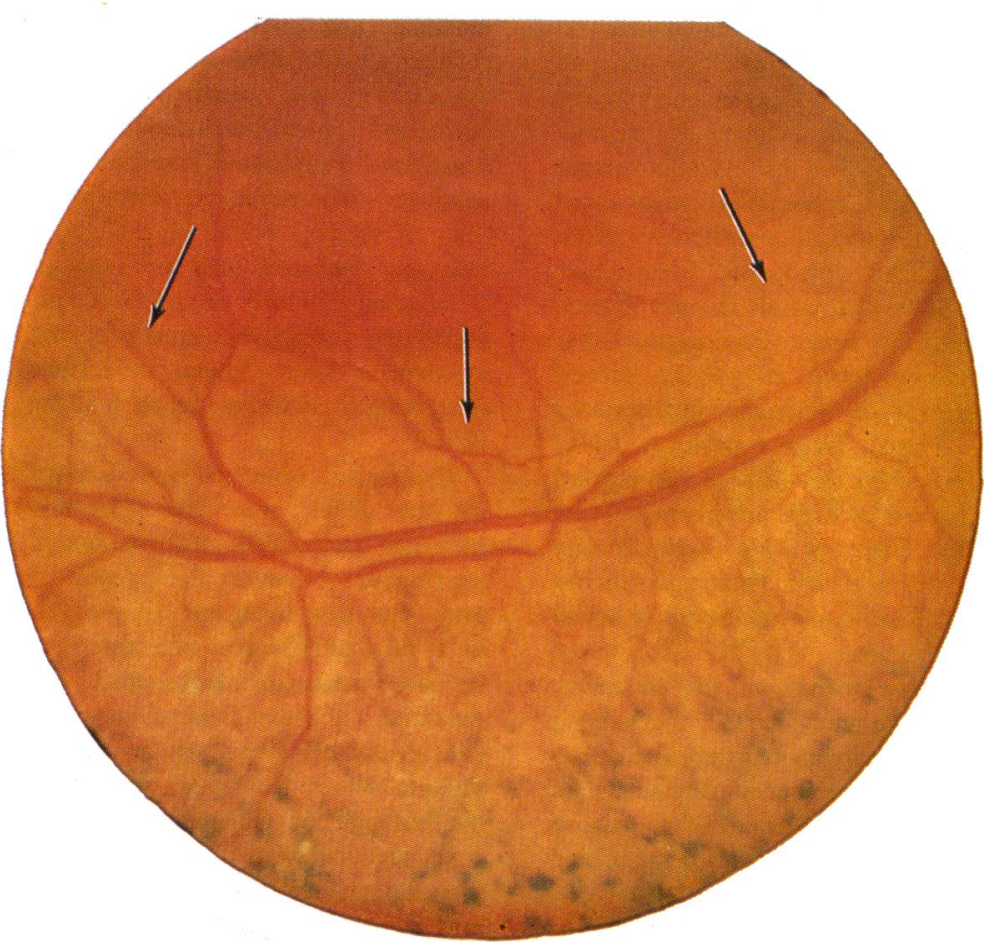

FIG. 9 Case 3.

Posterior extension of schisis to within inferior vascular arcade (arrows) 
reported by Blanck and others (1973). Implicit time values were not cited. In three cases reported by François and others (1974) photopic responses of either normal or subnormal amplitudes were noted. Photopic implicit times were prolonged in two of the three cases. Scotopic recordings showed either absent or much decreased b-wave amplitudes. Scotopic a-waves were recordable but reduced in amplitude. In our two younger patients, on whom ERG responses were still recordable, photopic and scotopic amplitudes were essentially identical. The photopic responses were either normal or subnormal in amplitude and prolonged in implicit time. Prolonged cone (photopic) implicit times have also been noted in most genetic patterns of retinitis pigmentosa (Berson and Kanters, 1970). In our patients ERG rod responses either failed to develop or developed minimally after 15 minutes of dark adaptation. The scotopic responses in Fig. $3^{b}$ are presumably predominantly from cone activity, thus accounting for the almost identical responses under both lightand dark-adapted conditions. Highly abnormal flicker fusion frequency responses in the oresence of normal or slightly subnormal ERG single-flash photopic amplitudes were an unexpected finding in two of the three cases. This discrepancy between single-flash photopic amplitudes and flicker fusion frequency has not been previously emphasized and may be characteristic of this disease.

Two reports of fluorescein angiographic findings in patients with Favre-Goldmann syndrome made no mention of either leakage from or non-perfusion of retinal vessels (MacVicar and Wilbrandt, 1970; Stankovic and others, 1973). The diffuse vascular leakage noted in our patients is unique among retinal dystrophies and has not been reported in association with peripheral or macular changes in $\mathrm{X}$-linked juvenile retinoschisis. Isolated reports have described perifoveal capillary leakage and cystoid macular oedema in patients with retinitis pigmentosa (ffytche, 1972; Gass, 1970). Since cystoid macular oedema was present in varying degrees in all three of our patients we suspect that at least some previously reported cases of Favre-Goldmann syndrome with 'macular schisis' may have had microcystic oedema from leaking perifoveal capillaries.

Dendritic opaque vessels similar to those seen in our cases have been noted in patients with juvenile X-linked retinoschisis. We observed a correlation between the presence of schitic changes and the development of vessel opacity, leakage, and non-perfusion. No retinoschisis was present in the posterior pole and only subtle opaque vascular changes were noted on contact lens examination. Even these mildly opaque retinal vessels were, however, incompetent, as evidenced by leakage of fluorescein. Moderate retinoschisis was present within the mid-peripheral retina in association with marked opaque vascular changes and extensive leakage of fluorescein dye. In areas 
with the most extensive schitic changes retinal vessels no longer appeared opaque but were nonperfused. A cause and effect relationship between non-perfusion and opaque retinal vessels and the development of retinoschisis cannot be supported with certainty by our findings. However, possibly those areas with extensive ischaemia of the inner retina are most susceptible to the development of schisis. An equally tenable explanation might implicate the schisis as causing secondary nonperfusion of retinal vessels. Other forms of retinoschisis do not cause a similar diffuse vascular leakage, and therefore another sequence of events (vascular leakage causing perivascular fluid accumulation and thereby retinoschisis) may apply to the Favre-Goldmann syndrome and may be analogous to the pathogenesis of cystoid macular oedema in this syndrome. In this disorder, as exemplified by case 2, ultimate occlusion of previously leaking vessels may result in a clinical situation characterized by maximum raising of schitic areas with absence of leakage (owing to total non-perfusion of previously involved vessels).

A diagnosis of Favre-Goldmann syndrome should be considered in patients presenting with an early history of poor night vision, bilateral atypical pigmentary changes of the retina, and degenerative changes in the vitreous humour. Additional diagnostic findings include retinoschisis, opaque retinal vessels, diffuse leakage from retinal capillaries, and cystoid macular oedema. Electroretinographic responses are either non-detectable or, if present, may show almost identical responses under both lightand dark-adapted conditions. In addition, highly abnormal flicker fusion frequency responses in the presence of relatively normal single-flash photopic amplitudes may be seen.

\section{Summary}

Extensive retinal vascular disease was noted in three patients from two families with FavreGoldmann syndrome. In addition to classical features they had pronounced leakage from some retinal vessels. Vessels were either opaque ('sclerotic') or non-perfused. Cystoid macular oedema was a contributing cause of decreased vision. Two of the three patients showed a discrepancy on electroretinography between single-flash photopic amplitudes and flicker fusion frequency. This may be characteristic of Favre-Goldmann syndrome.

This investigation was supported in part by the National Retinitis Pigmentosa Foundation, training grant EY24-I6 from the National Eye Institute, and an unrestricted award from Research to Prevent Blindness, Inc.

\section{References}

Berson, E. L., and KANTERS, L. (1970) Arch. Ophthal. (Chic.), 84, 288

BLANCK, M. F., POLlIOT, L., and BERNARD, P. (I973) Bull. Soc. franç. Ophtal., 86, 242

CARR, R. E., and SIEgel, I. M. (1970) Arch. Ophthal. (Chic.), 84, 436

FAVRE, M. (1958) Ophthalmologica (Basel), r35, 604

FFYTCHE, T. J. (1972) Trans. ophthal. Soc. U.K., 92, 265

FRANÇOIS, J., DE ROUCK, A., and CAMBIE, E. (1974) Ophthalmologica (Basel), I68, 81

- and VAN OYE, R. (1967) Ann. Oculist. (Paris), 200, 664

GASS, J. D. M. (1970) 'Stereoscopic Atlas of Macular Disease', p. I 54. Mosby, St Louis

MACVICAR, J., and WilbrandT, H. (1970) Arch. Ophthal. (Chic.), 83, 629

maraini, G. (1967) Ann. Ottal., 93, 343

RICCI, A. (1960) Ophthalmologica (Basel), 139, 338

STANkovic, I. v., KECMANovic, z., and DRINCIC, v. (I973; Bull. Soc. franc. Ophtal., 86, 246 